\title{
Anwendungen für Mehrphasen-CFD am Fahrzeugexterieur
}

\author{
Daniel Demel ${ }^{1,2}$ (D) $\cdot$ Thomas Schütz $^{1,3} \cdot$ Christian Kölzer $^{4} \cdot$ Cameron Tropea $^{1}$
}

Eingegangen: 30. Oktober 2020 / Angenommen: 13. März 2021 / Online publiziert: 9. April 2021

(c) Der/die Autor(en) 2021

\section{Zusammenfassung}

In der Automobilindustrie werden einphasige Strömungssimulationen seit vielen Jahren zur Aerodynamik- und Aeroakustikentwicklung eingesetzt. Sie gewähren einen detaillierten Einblick in das Strömungsfeld und helfen bei der Optimierung des Luftwiderstands und des Innenraumpegels in frühen Phasen des Fahrzeugentwicklungsprozesses. Daneben spielen zweiphasige Strömungssimulationen eine zunehmende Rolle, z.B. bei der Funktion der Scheibenwischer, der Sichtfreihaltung von Scheiben, der Reinigung von Sensoren oder dem Abtropfen von Regenwasser beim Öffnen von Türen und Klappen. Solche Mehrphasenströmungen sind jedoch weitaus komplexer und weniger gut verstanden. BMW arbeitet zusammen mit der TU Darmstadt an der Weiterentwicklung von Simulationsmethoden zur frühzeitigen Analyse und Auslegung des externen Wassermanagements.

Im vorliegenden Beitrag werden drei Anwendungsfälle mit zweiphasigen Strömungen betrachtet. Der erste Anwendungsfall umfasst die Beaufschlagung der Frontscheibe mit Regenwasser, dessen Verdrängung durch die Scheibenwischer und das Abfließen des Wassers. Beim zweiten Anwendungsfall wird das Ablaufverhalten von Regenwasser am stehenden Fahrzeug berechnet und überprüft, ob und wie das Wasser beim Öffnen der Heckklappe in den Fahrzeuginnenraum abtropft. Der letzte Anwendungsfall betrifft die Reinigung von Sensoren zur Fahrzeugumfeldüberwachung im Rahmen des teil-, hoch- und vollautomatisierten Fahrens. Damit das Reinigungsmedium jederzeit effizient seinen Zweck erfüllt, wird das Sensorreinigungssystem simulativ optimiert. Für alle drei Anwendungsfälle werden die zugrundeliegenden Methoden erläutert, Simulationsergebnisse präsentiert und ein Abgleich mit experimentellen Daten zur Validierung durchgeführt.

\section{Applications for multiphase CFD on vehicle exteriors}

\section{Abstract}

In the automotive industry single-phase flow simulations have been used during the aerodynamic and aeroacoustic development for years. They provide a detailed insight into the flow field and help to optimize drag and interior noise level in early stages of the vehicle design process. However, two-phase flow simulations play an increasing role, e.g. to optimize the function of windshield wipers, the wetting of windows, the cleaning of sensors or the dripping of rainwater while opening doors and flaps, and these flows are much more complex and less understood. BMW is working together with TU Darmstadt on the improvement of simulation methods for early analysis and design of external water management.

In this article three applications involving two-phase flows are considered. The first application pertains to the impact of rainwater on the windshield, its displacement by the wipers and the draining of the water. In the second application, the drainage behavior of rainwater on a stationary vehicle is studied, in particular whether and how the water drips into the vehicle interior when the tailgate is being opened. The last application concerns the cleaning of sensors which monitor the surroundings of a vehicle in the context of partially, highly and fully automated driving. To ensure that the cleaning fluid efficiently fulfils its purpose at all times, the sensor cleaning unit is optimized using simulations. For all three applications the underlying methods are explained, simulation results are presented, and a comparison with experimental data for validation is performed.

Daniel Demel

daniel.demel@bmw.de

1 Strömungslehre und Aerodynamik, TU Darmstadt, Alarich-Weiss-Str. 10, 64287 Darmstadt, Deutschland
2 BMW AG, Knorrstr. 147, 80788 München, Deutschland

BMW AG, Am Juliusturm 14-38, 13599 Berlin, Deutschland

4 EDAG Engineering, Emmy-Noether-Ring 6, 85716 Unterschleißheim, Deutschland 


\section{Einleitung und Anwendungslandkarte}

Zweiphasige Strömungsprobleme spielen bei der Entwicklung des Fahrzeugexterieurs eine bedeutende Rolle und beeinflussen sowohl sicherheits- als auch komfortrelevante Funktionen und Eigenschaften von Pkw. Dieses unter dem Begriff des externen Wassermanagements vereinte Themengebiet umfasst sämtliche Interaktionen von Wasser mit dem Fahrzeug. Dazu zählen:

- die Sicherstellung der Sichtfreihaltung in jeder Fahrsituation durch aktive Methoden wie die Auslegung des Scheibenwischersystems und passive Methoden wie Wasserfang- und -führungskonzepte,

- das Abfließverhalten des Wassers durch Nassbereiche des Motorraums sowie von Türen und Klappen während deren Öffnung,

- die Karosseriedichtheit bei starkem Regen sowie Folgeerscheinungen, wie Korrosion,

- die Platzierung und Reinigung von Sensorsystemen,

- die Belastungen der Fahrzeugfront mit Luftklappensystem und Rohluftansaugung sowie des Unterbodens bei einer Wasserdurchfahrt und

- die Benetzung von technisch relevanten Bereichen, wie Bremsen, Sensoren oder Türgriffen und Klappenbetätigungen.

Die Entwicklungsarbeit erfolgt heute zum überwiegenden Teil im Rahmen von Straßenfahrten oder im Umweltwindkanal, in dem verschiedene Schlechtwetterszenarien nachgestellt werden können. Da für beide Optionen ein physischer Prototyp vorhanden sein muss, finden solche Tests erst spät im Entwicklungsprozess statt. Erkannte, notwendige Änderungen sind zu diesem Zeitpunkt bereits sehr teuer. Eine umfassende Berücksichtigung der Wasserführung am virtuellen Fahrzeug mit numerischen Werkzeugen ist deshalb dringend erwünscht. Trotz der in den letzten Jahrzehnten enorm gestiegenen Computerrechenleistung stellt die Simulation von Mehrphasenströmungen noch immer eine Herausforderung dar.

BMW setzt bereits für einige solcher Problemstellungen numerische Strömungssimulationen (CFD) ein und arbeitet darüber hinaus gemeinsam mit der TU Darmstadt an validierten Methoden zur virtuellen Darstellung der oben genannten Probleme, vgl. [1]. Dabei kommen verschiedene Software-Lösungen zur Berechnung der Strömungsvorgänge zum Einsatz. Abb. 1 zeigt eine Übersicht zweiphasiger Strömungsprobleme am Pkw, bei denen BMW bereits CFDSimulationen in der Fahrzeugentwicklung einsetzt oder zurzeit aktiv an geeigneten Methoden arbeitet.

$\mathrm{Zu}$ den hervorgehobenen Fällen in Abb. 1 konnten in den vergangenen Monaten Fortschritte im Rahmen diverser Validierungsstudien erzielt werden. Eine Auswahl der
Fortschritte wird im vorliegenden Beitrag näher betrachtet. Tab. 1 fasst die ausgewählten Anwendungsfälle zusammen.

\section{Theorie und ausgewählte Verfahren}

Im ersten Anwendungsfall wird die Luftphase mit der Improved Delayed Detached-Eddy Simulation [2,3] unter Verwendung des $k-\omega$-SST-Turbulenzmodells nach Menter [4] berechnet. Zur Bestimmung der Turbulenz werden zwei zusätzliche Gleichungen für die turbulente kinetische Energie $k$ und die Dissipationsfrequenz $\omega$ gelöst. Die Abbildung der realitätsnahen Scheibenwischerbewegung in einer numerischen Simulation stellt u.a. aufgrund der komplex gekrümmten Frontscheibengeometrie eine Herausforderung dar. Abgesehen von Spezialfällen gibt es drei Möglichkeiten zur Darstellung von Netzbewegungen:

- Netzverformung,

- Neuvernetzung und

- Chimera-Netze.

Für die Abbildung der Scheibenwischerbewegung werden Chimera-Netze mit einer Netzverformung kombiniert. Bei Chimera-Netzen überlappen sich zwei Regionen, die unabhängig voneinander vernetzt werden. Die OversetRegion bewegt sich in der Hintergrundregion, während die Kopplung beider Regionen über Interpolation an den Berandungsflächen der Overset-Region erfolgt. Mit dieser Netzbewegungsmöglichkeit lassen sich große Distanzen bei gleichbleibender Gitterqualität umsetzen. Durch zusätzliche Netzverformung wird das Scheibenwischerblatt an die Frontscheibe angepasst.

Die Wasserphase wird mit dem hybriden Fluidfilm-VOFModell berechnet, das die Volume-of-Fluid-Methode (VOFMethode) mit dem Filmmodell koppelt. Durch Integration der Erhaltungsgleichungen für Masse und Impuls in Wandnormalenrichtung wird ein Gleichungssystem für die Filmhöhe $h_{f}$ und die beiden mittleren Geschwindigkeitskomponenten tangential zur Oberfläche $u_{i}$ hergeleitet, das das zweidimensionale Filmmodell beschreibt [5]:

$\frac{\partial \rho h_{f}}{\partial t}+\frac{\partial \rho h_{f} u_{i}}{\partial x_{i}}=S_{\rho \mathrm{h}}$
$\frac{\partial \rho h_{f} u_{i}}{\partial t}+\frac{\partial \rho h_{f} u_{i} u_{j}}{\partial x_{j}}=-\frac{\partial p h_{f}}{\partial x_{i}}+S_{\rho \mathrm{\rho hu}, i}$

Darin ist $\rho$ die Dichte des Fluids, $S_{\text {oh }}$ der Massenquellterm pro Wandflächeneinheit und $S_{\text {phu, } i}$ der Impulsquellterm. Der Einfluss durch die Oberflächenspannung fließt über den Kapillardruck $p_{\sigma}$ und die Kontaktlinienkraft $\tau_{\theta, \mathrm{i}}$ über Gln. (2a) und (2b)

$p_{\sigma}=-\alpha \sigma \frac{\partial}{\partial x_{i}}\left(\frac{\partial h_{f}}{\partial x_{i}}\right)$ 


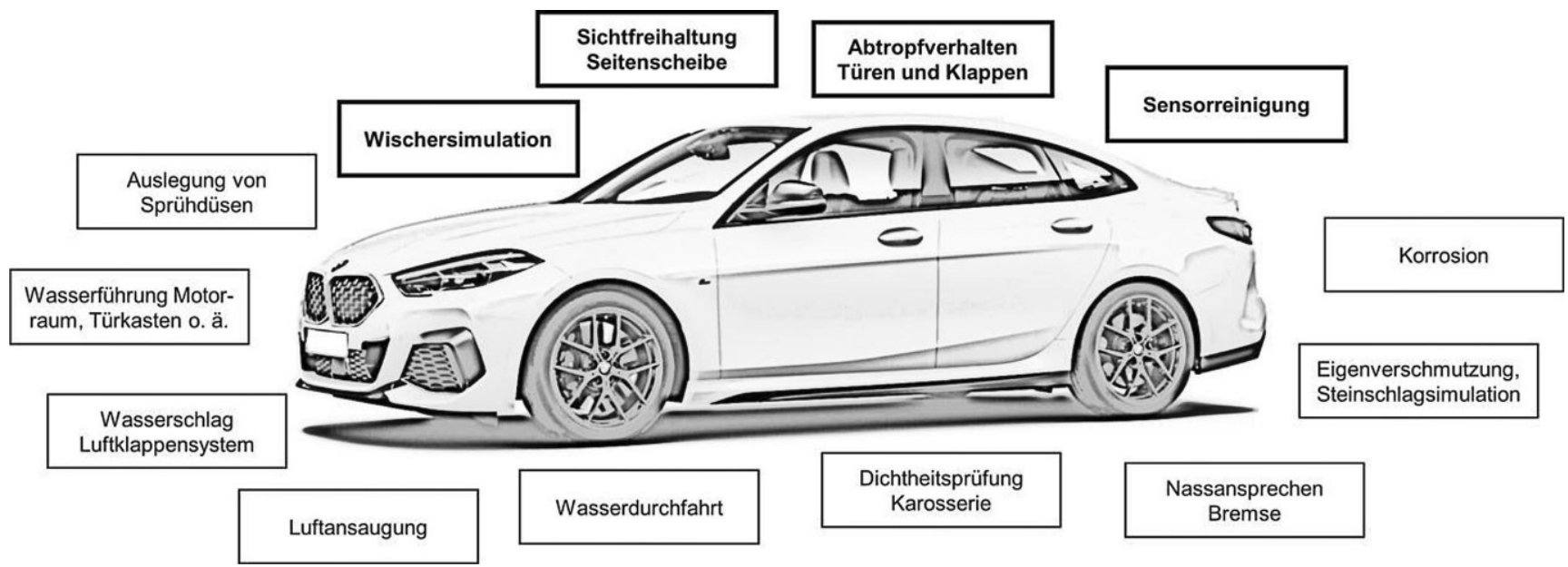

Abb. 1 CFD-Anwendungslandkarte mit Beispielen von zweiphasigen Strömungsvorgängen aus der Entwicklung des Fahrzeugexterieurs und Auswahl der diskutierten Lastfälle $(f e t t)$

$\tau_{\theta, i}=\beta \sigma \frac{1-\cos (\theta)}{l_{\mathrm{cl}}} n_{\mathrm{cl}, i}$

in das Gleichungssystem ein. Hierbei ist $\sigma$ der Oberflächenspannungskoeffizient des Fluids, $\alpha$ und $\beta$ Skalierungsfaktoren, $l_{\mathrm{cl}}$ die Länge des Kontaktlinienelements, $n_{\mathrm{cl}, i}$ der Einheitsnormalenvektor senkrecht zur Kontaktlinie und $\theta$ der Kontaktwinkel auf der jeweiligen Oberfläche. Außerdem wird die Wirkung der umliegenden Strömung auf den Fluidfilm mit einem Luftwiderstandsmodells berücksichtigt. Die Widerstandskraft wird dazu gemäß Gl. (3)

$\tau_{d, i}=\frac{1}{2} \rho_{L} c_{w} A_{s} u_{i}^{2}$

abgeschätzt und übergeben. Weitere Informationen zum Filmmodell sind der Literatur von Meredith [5] zu entnehmen.

In Regionen mit hohen Genauigkeitsanforderungen wird die zweite Phase mit der VOF-Methode berechnet. Die VOF-Methode zählt zu den Interface Capturing Verfahren. Neben den Erhaltungsgleichungen wird eine zusätzliche Gleichung für den Volumenanteil $\alpha$ gelöst. Ist der Volumenanteil einer Zelle 1, so ist diese vollständig mit der Phase gefüllt, ist dieser 0, dann enthält die Zelle die Phase nicht. An der freien Oberfläche zwischen Luft- und Wasserphase liegen die Volumenanteile zwischen 0 und 1. Die VOF-Methode benötigt grundsätzlich eine hohe zeitliche und räumliche Auflösung, um die freie Oberfläche korrekt aufzulösen, weshalb die Methode sehr rechenzeitintensiv und für große Simulationsregionen meist ungeeignet ist. Abhilfe schafft das hybride Mehrphasenmodell, bei dem der Wechsel zwischen beiden Modellen über einen vorgegebenen Grenzwert für den Volumenanteil $\alpha_{\text {trans }}$ gesteuert wird.

Beim zweiten Anwendungsfall wird PreonLab von Fifty2 eingesetzt. Es basiert auf dem Verfahren der Smoo- thed Particle Hydrodynamics (SPH) nach Gingold und Monaghan [6]. Die SPH ist ein effizienter, gitterloser und partikelbasierter Ansatz zur Fluiddynamik, der auf einer Approximation der Navier-Stokes Gleichungen für inkompressible Strömungen beruht. Das Fluid wird hierbei mit mehreren Partikeln diskretisiert, die die Masse $m$, das Volumen $V$, die Dichte $\rho$, die Position $x_{i}$ und die Geschwindigkeit $u_{i}$ besitzen. Die Partikel können sich frei im Raum bewegen. Die physikalischen Größen an einer Position werden über benachbarte Partikel unter Anwendung sog. Kernelfunktionen bestimmt. In Abhängigkeit des Einflussradius des Kernels werden dazu mehr oder weniger Nachbarpartikel berücksichtigt, wobei die Gewichtung mit zunehmendem Abstand von der betrachteten Position abnimmt.

Da PreonLab ein gitterloser Löser ist, können Geometrien ohne die zuvor aufgeführten Netzbewegungsmöglichkeiten bewegt werden. Die Luftphase wird über ein zugeschaltetes Modell berücksichtigt. Dieses Modell basiert auf der allgemeinen Formel zur Luftwiderstandskraft in Gl. (4) mit der Luftdichte $\rho_{L}$, der Querschnittsfläche der Partikel $A_{P}$, der Relativgeschwindigkeit $u_{\text {rel }}$ zwischen Luft- und Partikelgeschwindigkeit und dem Widerstandskoeffizienten $c_{w}$. Der Widerstandskoeffizient wird mit Hilfe des Liu-Modells [7] bestimmt.

$F_{L}=\frac{1}{2} \rho_{L} c_{w} A_{P} u_{\text {rel }}^{2}$

Effekte aufgrund von Oberflächenspannungen werden über Werte für Adhäsion und Kohäsion berücksichtigt. Die größten Vorteile der SPH Methode liegen in der Robustheit und der enormen Rechengeschwindigkeit.

Der dritte Anwendungsfall nutzt ein auf Lagrange Partikeln basiertes Verfahren in PowerFLOW von 3DS Dassault Systèmes. Die Strömung wird mit der auf der kinetischen Gastheorie aufbauenden Lattice-Boltzmann-Methode [8] 
Tab. 1 Ausgewählte Anwendungsfälle, die genutzte Simulationssoftware und eine kurze Beschreibung

\begin{tabular}{|c|c|c|c|}
\hline $\mathrm{Nr}$ & Anwendungsfall & Software & Beschreibung \\
\hline 1a & $\begin{array}{l}\text { Scheibenwischersimulation } \\
\text { und Frontscheibenbenet- } \\
\text { zung }\end{array}$ & $\begin{array}{l}\text { STAR-CCM+ } \\
\text { (Siemens) }\end{array}$ & $\begin{array}{l}\text { Darstellung der Scheibenwischerbewegung und Validierung der Frontscheiben- } \\
\text { benetzung während des Scheibenwischvorgangs ohne und mit Luftanströmung }\end{array}$ \\
\hline $1 b$ & $\begin{array}{l}\text { Sichtfreihaltung der } \\
\text { Seitenscheibe }\end{array}$ & $\begin{array}{l}\text { STAR-CCM+ } \\
\text { (Siemens) }\end{array}$ & $\begin{array}{l}\text { Abbildung des Wasserübergangs an der A-Säule aufgrund des von den Scheiben- } \\
\text { wischern zur A-Säule geschobenen Wassers und Betrachtung der resultierenden } \\
\text { Seitenscheibenbenetzung }\end{array}$ \\
\hline 2 & $\begin{array}{l}\text { Abtropfen beim Öffnen } \\
\text { von Türen und Klappen }\end{array}$ & $\begin{array}{l}\text { PreonLab } \\
\text { (Fifty2) }\end{array}$ & $\begin{array}{l}\text { Öffnen von Tür oder Heckklappe nach einer Beregnung und Betrachtung der in } \\
\text { den Innen- oder Gepäckraum abtropfenden Wassermenge an einem stehenden } \\
\text { Fahrzeug }\end{array}$ \\
\hline 3 & Reinigung von Sensoren & $\begin{array}{l}\text { PowerFLOW } \\
\text { (3DS Dassault } \\
\text { Systèmes) }\end{array}$ & $\begin{array}{l}\text { Betrachtung des Reinigungsvorgangs von Sensoren bei verschiedenen Fahrtge- } \\
\text { schwindigkeiten sowie Verfolgung des resultierenden Oversprays zur Überprü- } \\
\text { fung eines möglicherweise erneuten Auftreffens auf der Fahrzeugoberfläche }\end{array}$ \\
\hline
\end{tabular}

berechnet. Bei der kinetischen Gastheorie wird das Fluid durch molekulare Geschwindigkeitsverteilungsfunktionen beschrieben. Aus der Teilchenbewegung auf mikroskopischer Ebene werden makroskopische Fluidgrößen abgeleitet. Zur Turbulenzmodellierung wird in PowerFLOW ein VLES ähnliches Verfahren genutzt. Große Wirbel werden direkt berechnet und kleine, dissipative Wirbel werden mit der RNG-Form des $k$ - $\varepsilon$-Turbulenzmodells abgebildet [9]. Die zweite Phase, hier die Reinigungsflüssigkeit, wird über Sprühdüsen in das System eingetragen. Die eingesprühten Partikel sind massebehaftet und besitzen die Stoffeigenschaften (Dichte, dynamische Viskosität und Oberflächenspannung) des Reinigungsmediums. Die Sprühcharakteristik der Reinigungsdüsen wird individuell angepasst, sodass neben den gleichen Verteilungen für die Eintrittsgeschwindigkeiten und die Partikeldurchmesser auch der Volumenstrom und die Sprühkegelform und -größe wie im Experiment vorgegeben werden.

\section{Simulationsaufbau und Versuchsbeschreibung}

Bei Mehrphasensimulationen zur Scheibenwischerbewegung werden einige Funktionalitäten miteinander gekoppelt. Die Summe der Modelle und die hohe räumliche und zeitliche Auflösung der Regionen führen dazu, dass eine solche Simulation enorm aufwendig ist. Deshalb findet die Berechnung lediglich in einem reduzierten Ausschnitt statt. Für alle Oberflächen, auf denen ein Film erwartet wird, muss eine eigene Region, die sog. Shell, erzeugt werden. Darin können die Gleichungen des Filmmodells gelöst und die Filmgrößen gespeichert werden. Auf den Oberflächen wird jeweils der gemessene Kontaktwinkel in Form einer in Matlab berechneten, stochastischen Verteilung vorgegeben, um die Streuung des Kontaktwinkels einer realen Oberfläche zu berücksichtigen. Dieses Verfahren wurde in [1] erfolgreich getestet und führt zu einem realistischeren Benetzungsmuster.
Als Anfangs- und Randbedingungen werden die Strömungsfeldgrößen einer vorab berechneten Gesamtfahrzeugsimulation und eine Grundbenetzung vorgegeben, um die Erzeugung eines eingeschwungenen Zustands in der aufwendigen Simulation zu beschleunigen. Zur Validierung der Benetzung des Fahrzeugexterieurs unter Einfluss der Scheibenwischerbewegung wurde ein Validierungsversuch mit in die Simulation übertragbaren Randbedingungen konzipiert. Der schematische Aufbau an einer BMW 3er Limousine ist in Abb. 2 dargestellt. Vom extern befüllten Flüssigkeitsreservoir (grau) inkl. Überlauf wird die Flüssigkeit über eine Leitung (schwarz) mit Pumpe und Drosselventil zum auf dem Ausgleichsschaum (rot) platzierten Wasseraustrittskörper mit Beruhigungskammern (blau) geführt. Der Wasseraustrittskörper wird von hinten über mehrere auf der Breite verteilte Zuläufe mit Wasser befüllt und sorgt für einen möglichst gleichmäßigen und definierten Wassereintrag auf die Frontscheibe. Zur weiteren Verbesserung der Gleichmäßigkeit des Wasserfilms wird das Fahrzeug auf vier, individuell in der Höhe verstellbaren Stützen (dunkelgrün) aufgebockt. In einem iterativen Ausrichtprozess kann so ein gleichmäßiger Film auf der Frontscheibe realisiert werden. Zusätzlich wird ein vollständig durchtränktes Tuch über den Wasseraustrittskörper und den Ausgleichsschaum gelegt, das die Flüssigkeit von der Austrittsebene aufnimmt und auf die Scheibe abgibt. Im Bereich des Windlaufs wird das abfließende Wasser mit einem mehrstufigen Wasserfang- und -führungskonzept (hellgrün) aufgefangen und über Schläuche abgeführt. Damit lässt sich die durch den Motorraum fließende Wassermenge messen. Mit Kenntnis der zugeführten Wassermenge lässt sich der Anteil, der über die A-Säule befördert wird, bestimmen.

Die Validierungsversuche werden im Umweltwindkanal der BMW Group durchgeführt. Er ist wasserfest und abdunkelbar, sodass mithilfe der Fluoreszenzmethode die Benetzung auf dem Fahrzeug sichtbar gemacht werden kann. Dabei wird der Flüssigkeit ein geringer Anteil eines Fluoreszenzagenten beigemischt, der nach der Anregung mit ultraviolettem Licht spontan leuchtet. Das aufgenommene 
Abb. 2 Schematischer Versuchsaufbau des Validierungsexperiments im Umweltwindkanal (in Anlehnung an [17])

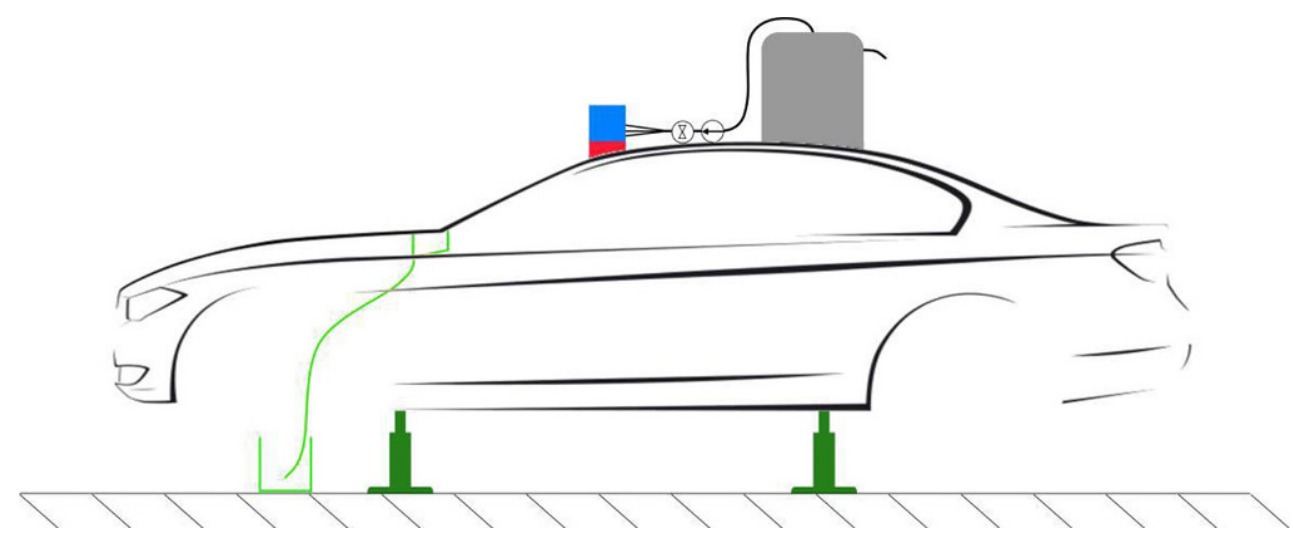

Abb. 3 Ablauf des Abtropfversuchs in der Dichtheitsprüfanlage (in Anlehnung an [16])

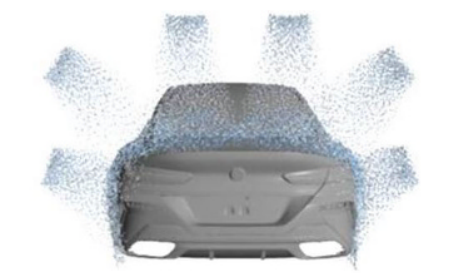

dreiminütige Beregnung mit einer Wasserbeaufschlagung von $25 \frac{1}{{\operatorname{min~} \mathrm{m}^{2}}^{2}}$
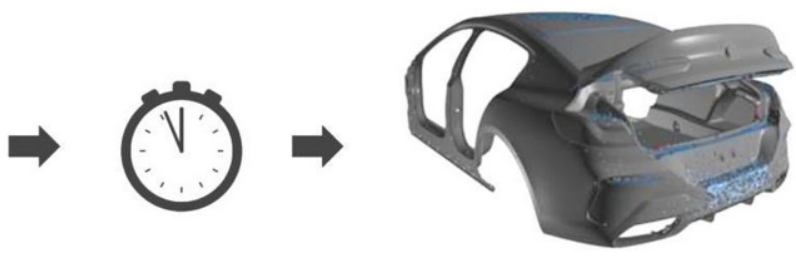

Wartezeit von einer Minute
Heckklappenöffnung und Beobachtung der Wasserpfade
Bildmaterial ist in Graustufen, wobei der Grauwert umso heller ist, je dicker die Filmschicht auf der Oberfläche ist. Durch vorherige Kalibriermessungen mit einer Höhenlehre bei gleichem Mischungsverhältnis von Fluoreszenzagent und Wasser und unverändertem Versuchsaufbau wird unter Verwendung des Durchstrahlungsgesetzes von Lambert [10] und Beer [11] eine Korrelation zwischen Grauwert und Filmhöhe hergeleitet (siehe auch [12]). Anschließend werden die Kameraaufnahmen in Abhängigkeit der Filmhöhe neu eingefärbt.

Die Abtropfversuche und -simulationen werden an zwei Fließheckfahrzeugen, einem BMW 8er Gran Coupé und einem 6er Gran Turismo, durchgeführt. Der Versuchsablauf zur Überprüfung des Abtropfverhaltens wird dem BMW Group Standard zur Kraftfahrzeugdichtheit gegen Wasser GS 97004-1 [13] und GS 97004-2 [14] entnommen und ist in Abb. 3 gezeigt. Bei der Standardprüfung wird das Fahrzeug in einer Dichtheitsprüfanlage drei Minuten lang einem Starkregenereignis mit einer Wasserbeaufschlagung von $25 \mathrm{l} / \mathrm{min} / \mathrm{m}^{2}$ ausgesetzt. Die anschließende Wartezeit von einer Minute vor Öffnung der Heckklappe bildet das Kundennutzungsverhalten ab und verhindert ein direktes Abtropfen aus den Beregnungsdüsen. Beim Öffnen der Heckklappe läuft das auf dem Fahrzeug und in den Auffangtaschen verbliebene Wasser ab und tropft möglicherweise in den Gepäckraum.

Grundvoraussetzung für reproduzierbare Messungen ist die Beibehaltung vergleichbarer Kontaktwinkel als Maß für die Benetzbarkeit der Oberfläche. Für den Kontaktwinkel der Heckklappe wird ein Bereich zwischen 70 und $75^{\circ}$ angestrebt. Um den Kontaktwinkel im genannten Bereich zu halten, wird die Oberfläche durch Polieren vorkonditioniert. Zur Kontrolle und Erfassung der Kontaktwinkel dient ein Kontaktwinkelmessgerät der Firma Krüss [15]. Nach Auftragung eines dosierten Tropfens auf die Oberfläche wird der Winkel zwischen Festkörper und Wassertropfen aus der Kameraaufnahme ermittelt. Vor jedem Versuch werden je zehn Messwerte an fünf verschiedenen Stellen der Heckklappe genommen.

Vor Beregnung des Fahrzeugs wird der Gepäckraum mit Papier ausgelegt. Dadurch können die beim Öffnen der Heckklappe abfallenden Wassertropfen lokalisiert und gezählt werden. Ihr Volumen wird durch Wägung des Papiers vor und nach dem Abtropfversuch bestimmt. Nach Ermittlung der praktischen Ergebnisse des Abtropfversuchs folgt die rechnergestützte Simulation in PreonLab mit einer möglichst realen Abbildung der Umgebung. Für die Simulation des Abtropfverhaltens sind das Fahrzeug mit seinen Oberflächeneigenschaften und die Beregnungsdüsen exakt zu integrieren. Nach aufwändiger Parameterstudie in [16] wird für die Simulation eine Partikelgröße von $1,5 \mathrm{~mm}$, eine deutlich verkürzte Beregnungsdauer von $7,5 \mathrm{~s}$ und eine Abtropfdauer von $30 \mathrm{~s}$ als sinnvoller Kompromiss aus Genauigkeit und Rechendauer ausgewählt.

Das Berechnungsmodell ist auf die Fahrzeugbauteile reduziert, die für die Wasserbeaufschlagung der Heckklappe relevant sind. Da in der PreonLab Version 3.3 Kontaktwinkel nicht explizit definiert werden können, wird für die 

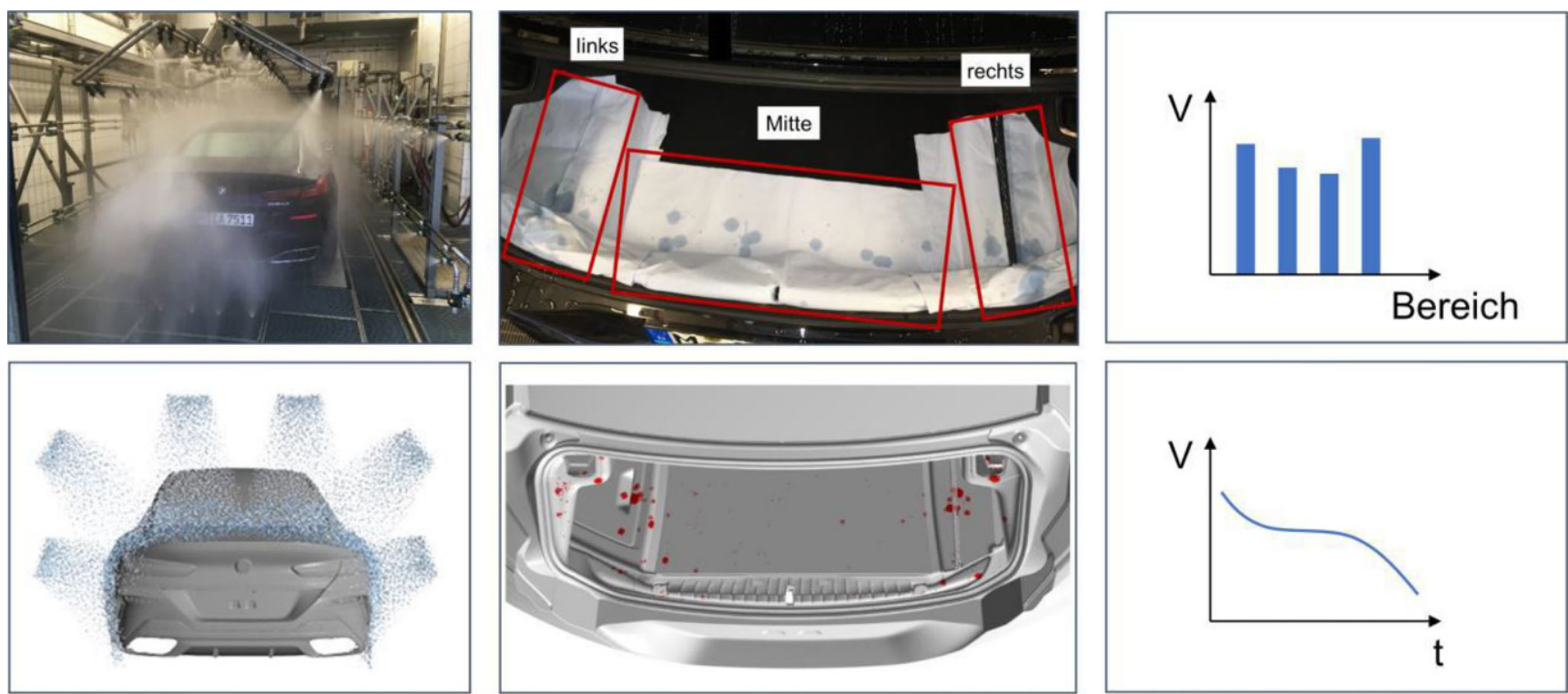

a) Beregnung

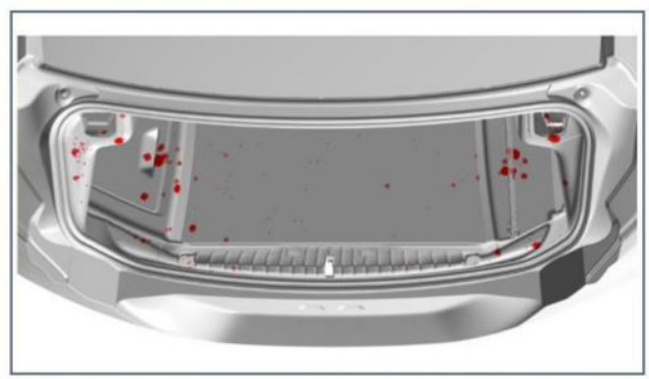

b) Abtropfen

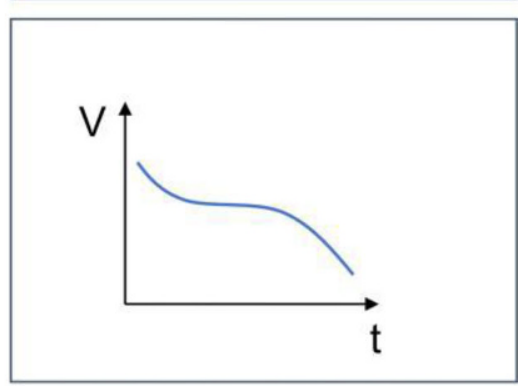

c) Ergebnis

Abb. 4 Simulation und Validierungsexperiment zum Heckklappenabtropfen (in Anlehnung an [16])

Adhäsion der Wert 0,65 und für die Kohäsion der Wert 1,8 eingestellt, um den im Versuch angestrebten Kontaktwinkelbereich zwischen 70 und $75^{\circ}$ zu modellieren. Die Werte sind dimensionslos und empirisch für das Verhalten von Wasser auf Glas ermittelt. Unter Beachtung des genannten Group Standards wurde die Dichtheitsprüfanlage mit allen relevanten Düsen nachgebildet. In der Simulation werden insgesamt sechs Reihen mit jeweils acht Düsen sowie eine Reihe mit senkrechten und eine Reihe mit angewinkelten Unterbodendüsen nachgebildet. Neben dem Abstand und der Ausrichtung wird der aus einem Foto ermittelte Austrittswinkel des Sprays mit $60^{\circ}$ vorgegeben. Die vorgeschriebene Wasserbeaufschlagung beträgt $251 / \mathrm{min} / \mathrm{m}^{2}$, woraus sich bei einer Fläche von $12 \mathrm{~m}^{2}$ und insgesamt 56 Düsen eine Wassermenge von ca. $5,41 / \mathrm{min}$ pro Düse ergibt. Als Vorgabe werden 6,01/min pro Düse eingestellt, um mit einer etwas stärkeren Wasserbeaufschlagung die im Vergleich zur Realität kürzere Simulationsdauer auszugleichen. [16]

Das Versuchsfahrzeug verfügt über eine automatische Heckklappenöffnung, wodurch sich die Klappe stets gleich schnell öffnet. Die Gesamtöffnungsdauer beträgt 3,5 s, wobei in den ersten und letzten 0,5 s die Beschleunigung bzw. Verzögerung stattfindet. Das Öffnungsverhalten wird in der Simulation durch einen quadratisch zunehmenden bzw. abnehmenden und einen linearen Verlauf abgebildet. Der schematische Ablauf von Versuch und Simulation ist in Abb. 4 dargestellt.

Für die Sensorreinigungssimulation mit PowerFLOW müssen die Sensorgeometrien inkl. der Reinigungsdüsen detailliert vernetzt und mit der Fahrzeugaußenhaut verschnitten werden. Ein Bild des Sensors inklusive Reinigungsdüsen und Sprühkegeln zeigt Abb. 5. Die Abbildung der Reinigungsdüsen und die korrekte Verortung am Versuchsfahrzeug, einem umgebauten BMW X3 Sport Utility Vehicle, sind entscheidend für das resultierende Benetzungsbild, da diese die lokalen Strömungsverhältnisse nahe der Sensoren beeinflussen. Weiterhin gilt es, den Sprühkegel sowie die Spraycharakteristik so realistisch wie

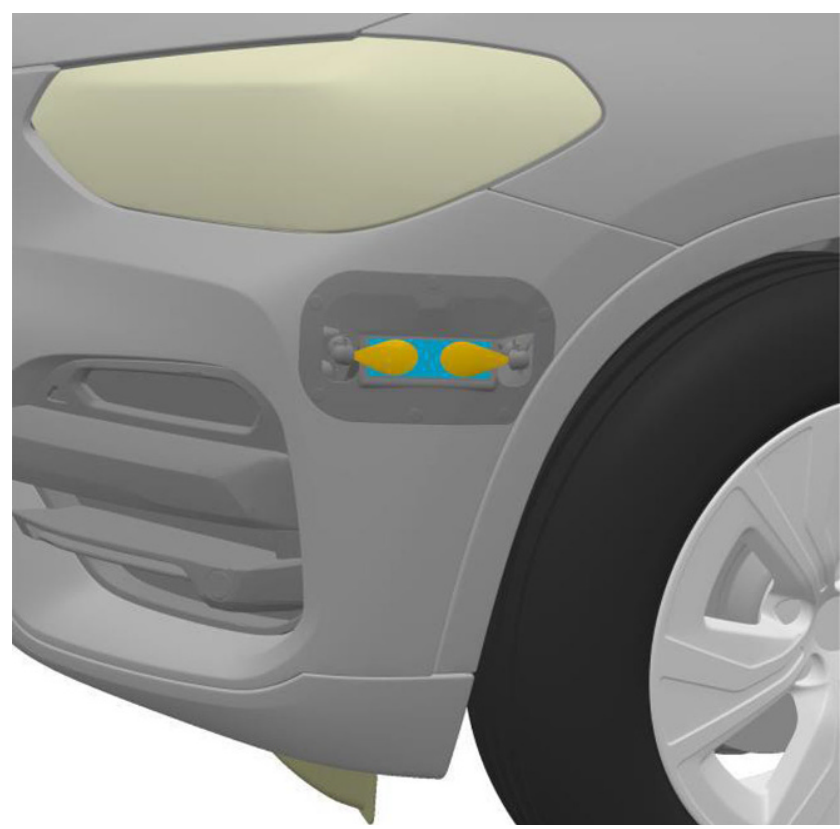

Abb. 5 Lidarsensor mit beidseitigen Reinigungsdüsen und Sprühkegeln in der linken Seite der Frontschürze eines Prototyps 


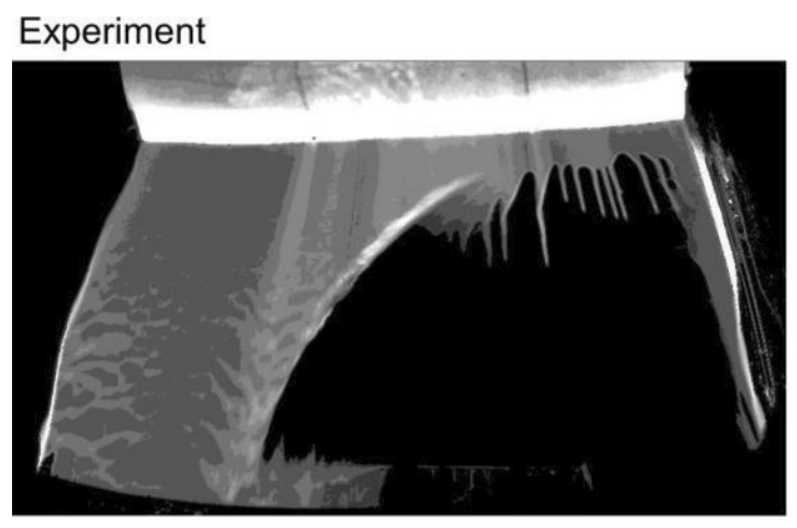

\section{Simulation}

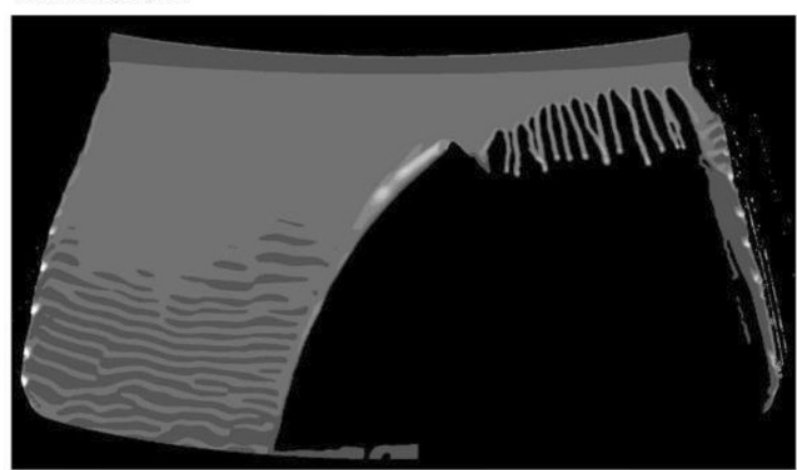

Filmhöhe in $\mathrm{mm}$

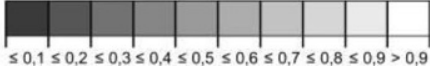

Abb. 6 Frontscheibenbenetzung während der Scheibenwischerbewegung in Experiment und Simulation (in Anlehnung an [17])

möglich abzubilden. Die Öffnungswinkel für beide Halbachsen des elliptischen Vollkegels sowie Kenngrößen des Sprays (Partikeldurchmesser und -geschwindigkeitsverteilung, Volumenstrom, Dichte, Viskosität, etc.) sind wichtige Randbedingungen für die Simulation. Als Reinigungsmedium wird Scheibenreiniger mit Frostschutz (BMW First Fill) eingesetzt.

Die Simulation zur Sensorreinigung ist ähnlich zu einer reinen Aerodynamiksimulation aufgebaut, wobei die Bereiche nahe des Sensors zusätzliche Verfeinerungsboxen aufweisen. Außerdem werden extra Regionen definiert, in denen zum einen die Flüssigkeit in den Zellen über einen selbst definierten Parameter sichtbar gemacht wird und zum anderen die Partikelbahnen verfolgt werden. Außerhalb dieser Regionen werden die Partikel entfernt, um Rechenkosten einzusparen. Vor Beginn des Reinigungsprozesses wird die einphasige Luftumströmung berechnet bis ein auskonvergierter Strömungszustand vorliegt. Danach wird die zweite Phase in Form des Flüssigkeitssprays der Reinigungsdüsen in die Simulationsdomain eingetragen und verfolgt. Dabei liegt eine Zweiwegekopplung vor, d.h. die Luftströmung beeinflusst die Partikel und umgekehrt. Die kontinuierliche Luftphase und die disperse Flüssigkeitsphase werden simultan berechnet.

\section{Ergebnisteil}

Abb. 6 zeigt die Gegenüberstellung von Experiment im Umweltwindkanal der BMW Group und Mehrphasensimulation mit Wasseraustrittskörper in der frontalen Ansicht. Qualitativ ist die Übereinstimmung sehr gut. Die linke Hälfte der Frontscheibe ist in beiden Fällen mit einem dünnen Film benetzt. Im oberen Bereich ist der Film glatt, während er mit zunehmender Lauflänge instabiler wird und sich Ober- flächenwellen ausbilden. Nachdem der Scheibenwischer die Benetzung in der rechten Hälfte der Frontscheibe beseitigt hat, erfolgt die Wiederbenetzung in Form von Rinnsalen, die ohne zu mäandrieren herabfließen. Die Rinnsale in der Simulation sind gleichmäßiger als im Experiment. Bei der Bewegung der Scheibenwischer wird das aufgesammelte Wasser durch Zentrifugalkräfte nach außen getrieben und führt zur Aufdickung des Films am Scheibenwischeraußenkreis. Die scheinbar sehr großen Filmhöhen nahe der A-Säule und auf dem Wasseraustrittskörper im Experiment resultieren aus einer Überschätzung der vorliegenden Intensität aufgrund des über den Austrittskörper gelegten Tuchs bzw. des zur Schließung von Spalten genutzten AluminiumKlebebands.

Die Untersuchungen wurden jeweils mit verschiedenen A-Säulenvarianten durchgeführt. Die A-Säulenvarianten unterscheiden sich in der Wasserfangleiste: Variante 1 besitzt zwei parallele Rinnen und Variante 2 ist rinnenlos. Bei Betrachtung der Seitenscheibenbenetzung von Variante $1-$ zu sehen in Abb. 7 - zeigt sich ebenfalls eine gute Übereinstimmung zwischen Simulation und Experiment. Die Scheibenwischer befördern Wasser in regelmäßigen Zeitabständen zur A-Säule. Da das ankommende Wasser nicht vollständig vom Wasserfangkonzept aufgenommen und abtransportiert werden kann, kommt es zum Übertritt an der A-Säule. Die Seitenscheibe wird sowohl im Experiment als auch in der Simulation jeweils im vorderen Drittel mit einzelnen Rinnsalen benetzt. Bei Variante 2 gelangt eine größere Wassermenge über die A-Säule und die Benetzung der Seitenscheibe erfolgt in Form eines flächigen Films.

Die gleichen Untersuchungen werden mit geringer Luftströmungsgeschwindigkeit wiederholt. Es wird eine geringe Geschwindigkeit gewählt, um ein Wegfliegen von nur leicht fixierten Teilen des Versuchsaufbaus zu vermeiden und um 

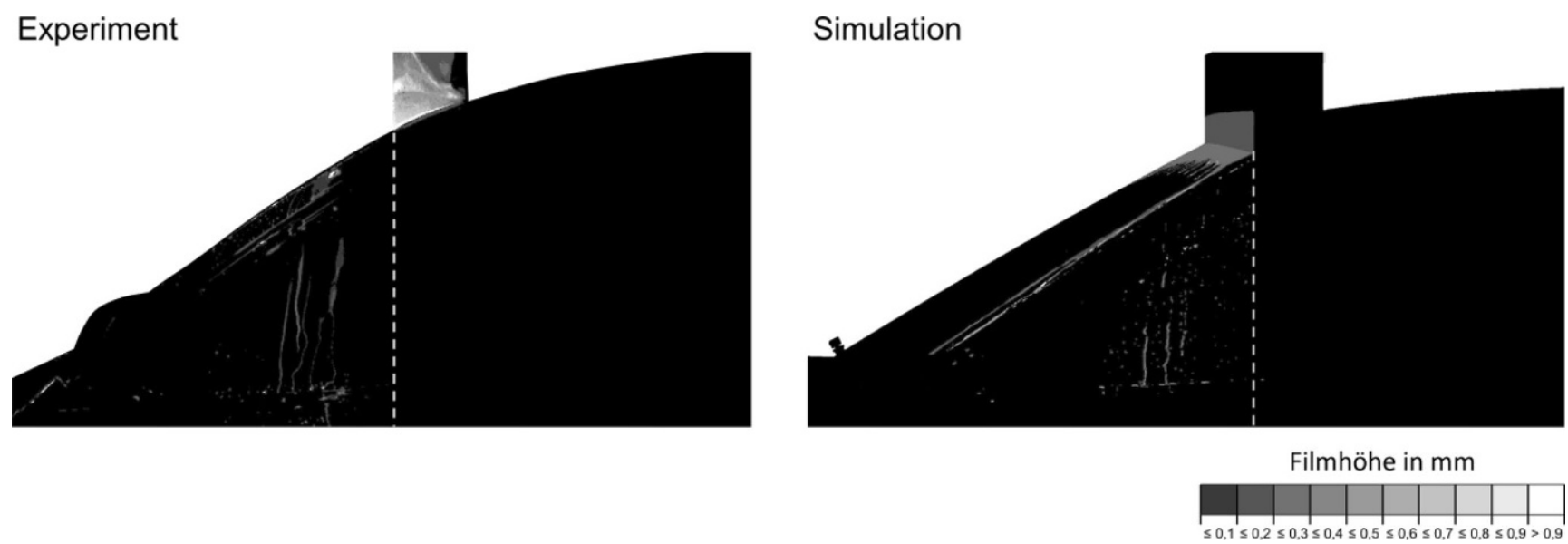

Abb. 7 Seitenscheibenbenetzung während der Scheibenwischerbewegung in Experiment und Simulation (in Anlehnung an [17])
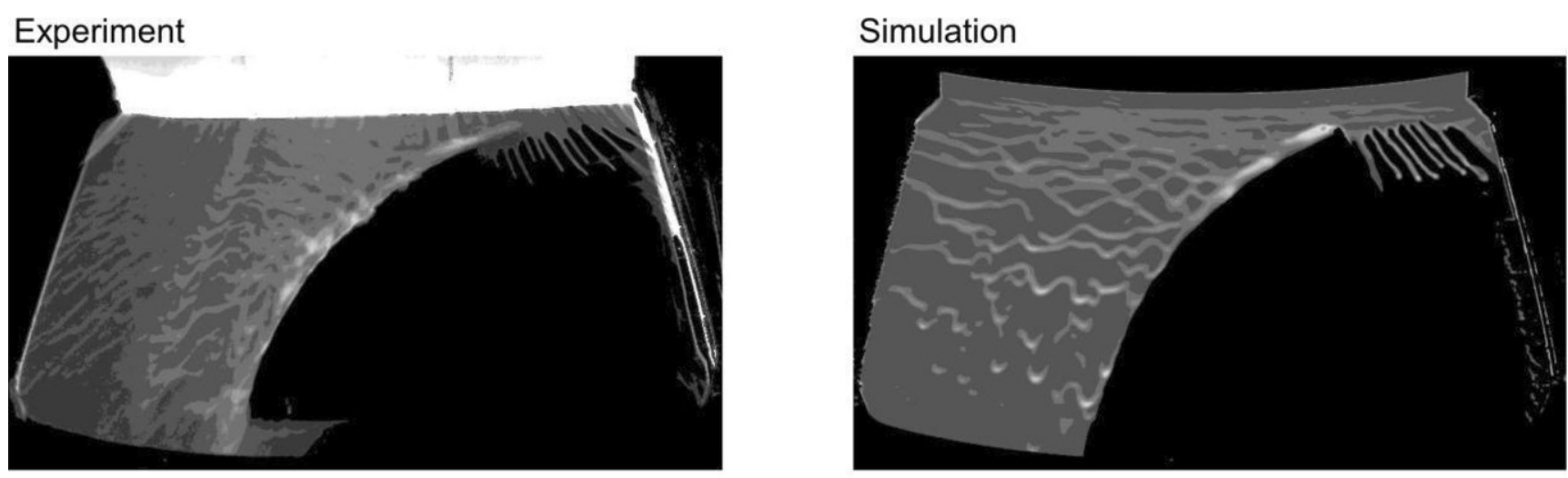

Filmhöhe in $\mathrm{mm}$

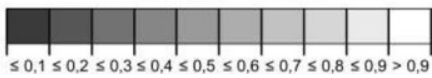

Abb. 8 Frontscheibenbenetzung während der Scheibenwischerbewegung in Experiment und Simulation unter Luftanströmung (in Anlehnung an [17])

zu gewährleisten, dass die Flüssigkeit auf der Frontscheibe herabfließen kann. Abb. 8 zeigt die Gegenüberstellung der Frontscheibenbenetzung unter Luftanströmung. Die linke Hälfte der Scheibe wird erneut mit einem flächigen Film benetzt. Im Gegensatz zu Abb. 6 bilden sich die Oberflächenwellen des Films nun deutlich früher und unregelmäßiger. In dem vom Scheibenwischer überfahrenen Ausschnitt der Frontscheibe fließen Rinnsale mit zusätzlicher nach außen gerichteter Querkomponente herunter. Je näher die Rinnsale an der A-Säule sind, desto stärker ist deren Ablenkung durch die Luftströmung.

Die Validierung der Simulation wird neben dem qualitativen Vergleich anhand von quantitativen Größen durchgeführt. Eine solche Größe ist das Verhältnis aus der über die A-Säule transportierten Wassermenge und der zugeführten Wassermenge. Der prozentuale Wasseranteil, der über die A-Säule befördert wird, ist für beide A-Säulenvarianten und jeweils mit und ohne Luftanströmung in Abb. 9 aufgeführt. Die blauen Balken mit den Fehlerbalken kennzeichnen die
Experimente und die orangen Balken die dazugehörigen Simulationen. Im Experiment werden die zugeführte und die durch den Motorraum fließende Wassermenge über einen längeren Zeitraum gemessen, während in der Simulation aufgrund der enormen Rechenzeit lediglich ein kompletter Scheibenwischerzyklus im eingeschwungenen Zustand herangezogen wird. Für alle untersuchten Varianten liegt der Wasseranteil der durchgeführten Simulationen innerhalb der etwa 5-7\%igen Streubreite der Experimente. Die Simulationen bilden somit sowohl die Änderung der A-Säulengestalt als auch die Erhöhung der Anströmgeschwindigkeit korrekt ab. [17]

Ergebnisse der Abtropfsimulation sind das Abtropfvolumen in den Kofferraum, die Tropfenverteilung und die Analyse des Wasserablaufpfads. Abb. 10 zeigt den Vergleich von Abtropfvolumen und Abtropfbild zwischen Experiment und Simulation. Das abtropfende Wasservolumen kann für beide Fahrzeuge mit etwa 7\%iger Abweichung vorhergesagt werden. Bei der Tropfenverteilung findet das 
Abb. 9 Prozentualer Wasseranteil, der über die A-Säule befördert wird, für verschiedene A-Säulenvarianten mit und ohne Luftanströmung (nach [17])
Abb. 10 Vergleich zwischen Simulation und Versuch zum Heckklappenabtropfen: Abtropfvolumen für beide Fahrzeuge (links) und Abtropfbild (rechts) (in Anlehnung an [16])
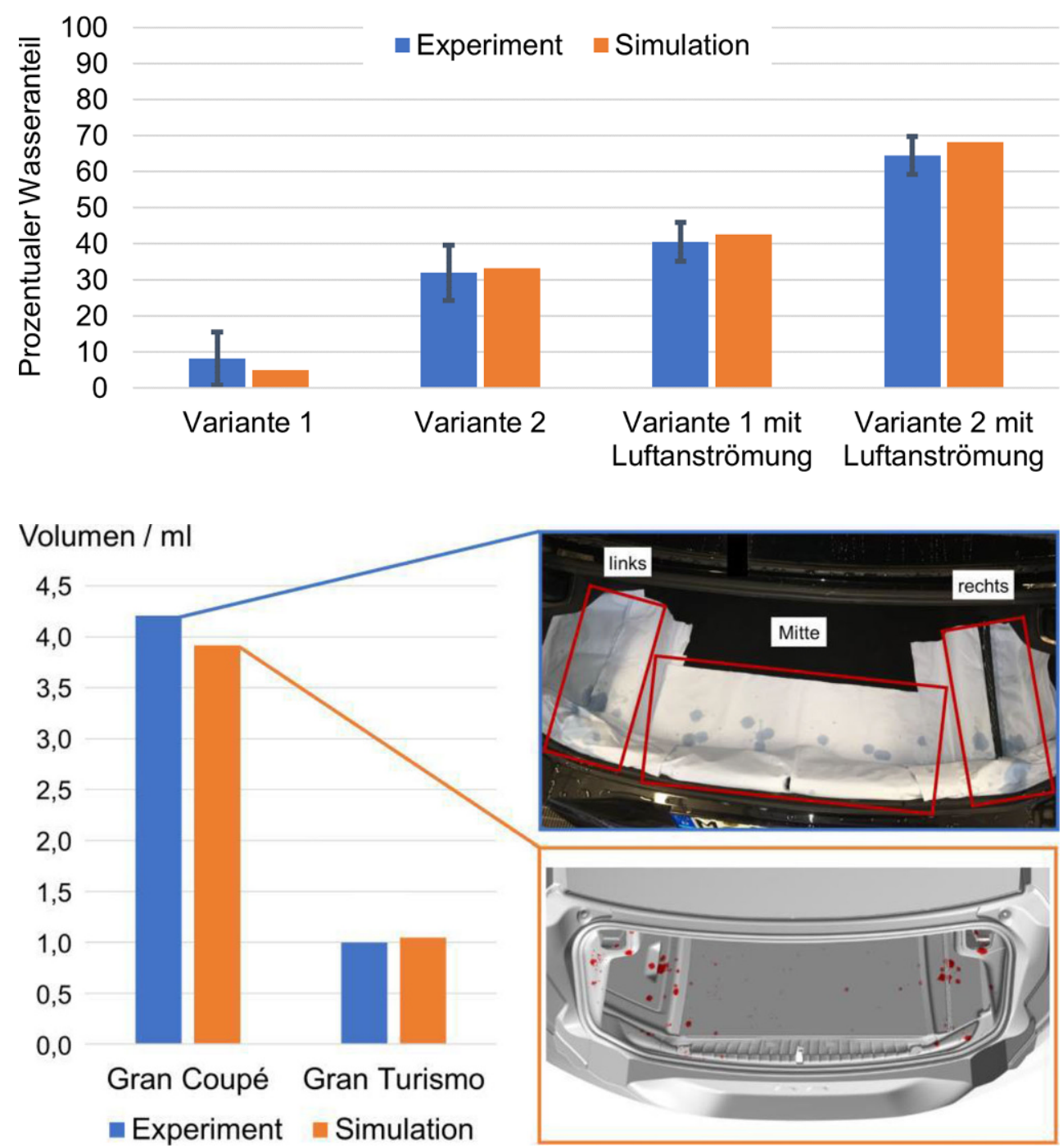

schwerpunktmäßige Abtropfen im seitlichen Bereich statt. Die Tropfen fallen in der Simulation allerdings in Fahrzeugrichtung weiter vorne in den Gepäckraum. Außerdem kommt es in der Simulation zu signifikant weniger Abtropfen im mittleren Bereich. Mögliche Ursachen sind die beschriebene Kontaktwinkelapproximation oder die in der Simulation nicht abgebildete Verformung sowie das Setzverhalten von Dichtungen.

Für die zukünftige Fahrzeugentwicklung ist das Nachvollziehen des Wasserablaufpfads von besonderem Wert. Dies ist in Abb. 11 für die in die Heckklappe integrierte Wasserfangleiste mit vier Phasenbildern bei klappenfestem Aufnahmewinkel während des Öffnungsvorgangs gezeigt. Zunächst sammelt sich Abtropfwasser aus Hohlräumen im Randbereich der Wasserfangleiste (Öffnungswinkel $37^{\circ}$ ). Beim weiteren Öffnen läuft das Wasser in der Fangleiste in Richtung des tiefsten Punkts und trifft dort auf das aus den Hohlräumen in die Fangleiste tropfende Wasser (Öffnungswinkel $45^{\circ}$ ). Da die Kapazität der Leiste nicht aus- reicht, um das Wasser zurückzuhalten, tropft es von dieser Stelle in den Kofferraum ab (Öffnungswinkel 57²). Zuletzt fließt das in der Fangleiste verbliebene Wasser zum neuen, tiefsten Punkt und tropft von dort ab (Öffnungswinkel $68^{\circ}$ ). Mit der Simulation können die Wasserpfade detailliert verfolgt werden, wohingegen eine solche Analyse in Versuchen nicht oder nur unter erheblichem Aufwand leistbar wäre. [16]

Der Fokus des dritten Anwendungsfalls liegt in der Ermittlung einer geeigneten Sensorposition, sodass einerseits die Funktion des Sensors sichergestellt und andererseits die Verschmutzung weitestgehend vermieden wird. Sollte sich der Sensor trotzdem mit Schmutz zusetzen, muss durch einen Reinigungsvorgang der saubere Grundzustand wieder hergestellt werden. In der nachfolgenden Untersuchung wird der Reinigungsvorgang eines Sensors in der Fahrzeugfront analysiert. Die durch Reinigungsdüsen auf die Sensorfläche gesprühten Flüssigkeitspartikel sollten im Idealfall neben dem Sensor keine anderen Stellen benet- 
Abb. 11 Analyse des Wasserablaufpfads an der Heckklappe mit Hilfe der numerischen Simulation (Ansichten klappenfest) (in Anlehnung an [16])

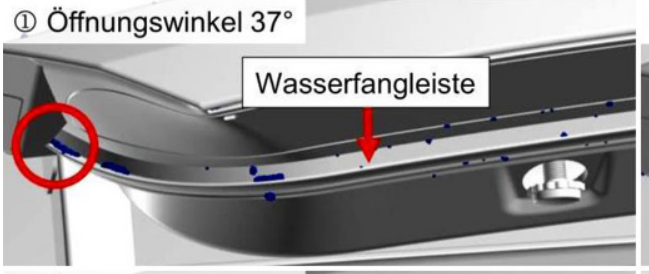

(2) Öffnungswinkel $45^{\circ}$
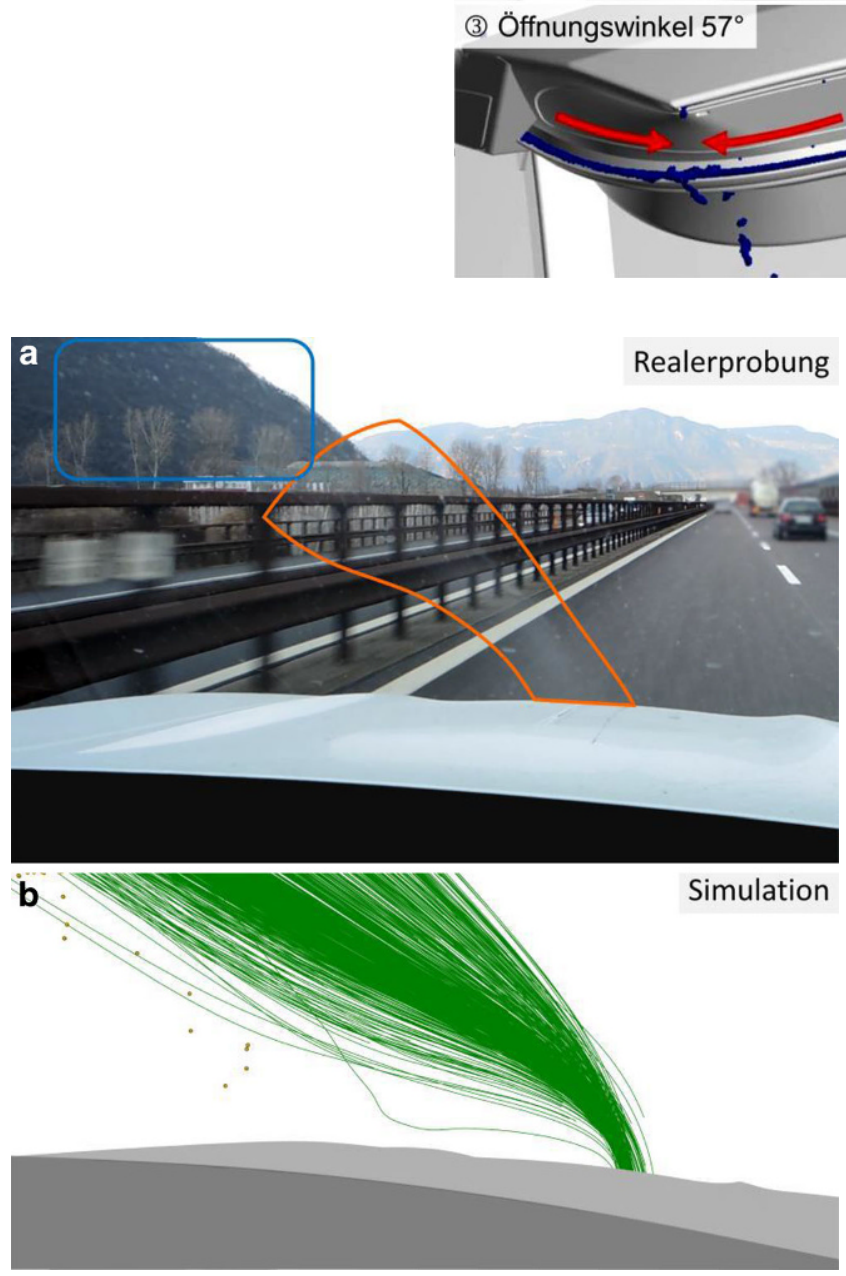

Abb. 12 Sprühnebel bei der Sensorreinigung und Auftreffpunkte der Reinigungsflüssigkeit auf der Frontscheibe aus Sicht vom Fahrzeuginneren durch die Frontscheibe (a Realerprobung, b Simulation)

zen. Per Simulation wird der Bewegungspfad der Partikel verfolgt und geprüft, ob sich diese während oder nach dem Reinigungsprozess auf anderen Flächen wie z. B. der Frontscheibe ablagern. Abb. 12 zeigt eine Gegenüberstellung des Reinigungsvorgangs der Kamera in der Frontschürze während der Realerprobung und in der Simulation.

Die Kamera ist mittig im oberen Bereich der Niere verortet und damit oberhalb des frontalen Staupunkts, wodurch ein Großteil der ablösenden Partikel mit der Strömung über die Frontklappe getragen wird. Dies ist in der Realerprobung anhand von Sprühnebel in der Luftströmung (orange eingerahmt) und in der Simulation durch die grünen Partikelbahnen zu erkennen. Vereinzelt lagern sich Flüssigkeit- spartikel im oberen Bereich der Frontscheibe ab. In der Realerprobung ist die betroffene Stelle blau umrahmt und in der Simulation sind die Auftreffpunkte der Partikel durch gelbe Kugeln repräsentiert. Die Partikelbahnen und die Auftreffpunkte auf der Frontscheibe in der Simulation stimmen mit den Ergebnissen aus der Realerprobung überein. Die numerische Partikelsimulation kann folglich zur Bestimmung einer geeigneten Sensorposition genutzt werden. Da bei der Wahl der Sensorverortung auch andere Aspekte, wie die Sicherstellung der Funktion, die Luftwiderstandsbeeinflussung oder das Design des Erscheinungsbildes, berücksichtigt werden müssen, kann eine geringfügige Benetzung einzelner Stellen nicht vollständig ausgeschlossen werden.

Ein weiteres Beispiel zur Sensorreinigung ist der in Abb. 5 aufgeführte Lidarsensor mit den beidseitig platzierten Reinigungsdüsen und Sprühkegeln in der linken, vorderen Schürze eines Prototyps. Die Untersuchungen sind in einer sehr frühen Phase durchgeführt worden, weshalb die Integration der Sensoreinheit in die Frontschürze nur behelfsmäßig zur Durchführung der Versuche umgesetzt wurde. In der ersten Version wird relativ viel Reinigungsflüssigkeit durch die lokalen Strömungsverhältnisse umgelenkt. Abb. 13 zeigt die übereinstimmenden Ergebnisse aus Versuch und Simulation zum Zeitpunkt der Reinigung durch die mit der Hauptströmung orientierten Sprühdüse. Das Reinigungsmedium trifft die Sensorfläche nur partiell. Ferner sind starke Verwirbelungen erkennbar, die ein Teil der Flüssigkeit sogar entgegen der Hauptströmungsrichtung transportieren.

Die berechnete Simulation ermöglicht eine detaillierte Analyse der vorliegenden Strömungsverhältnisse und offenbart, dass durch die Öffnungen in der Frontschürze um die Reinigungsdüsen Luft mit einer hohen Geschwindigkeitskomponente austritt, die das Reinigungsfluid mitreißt (vgl. Abb. 14a). Durch Schließung der um die Reinigungsdüsen vorliegenden Löcher in der Frontschürze werden die Strömungsverhältnisse verbessert und das Nachlaufgebiet hinter den Reinigungsdüsen verkleinert (vgl. Abb. 14b). Der Sensor wird effizienter mit einem niedrigeren Flüssigkeitsbe- 
Abb. 13 Gegenüberstellung der Sensorreinigung an der seitlichen Frontschürze (a Experiment, b Simulation) a

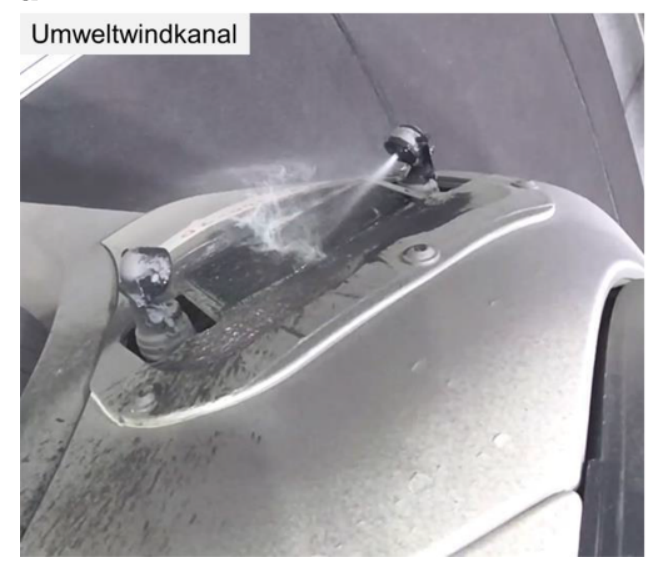

b

Simulation

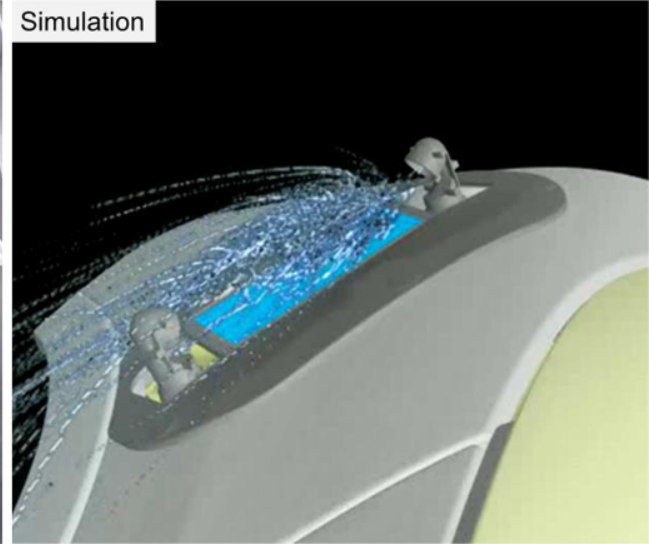

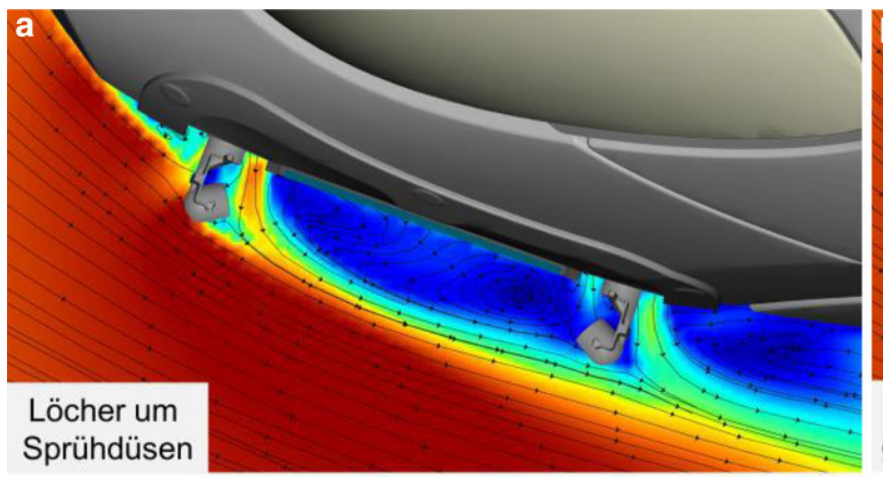

Löcher um

Sprühdüsen

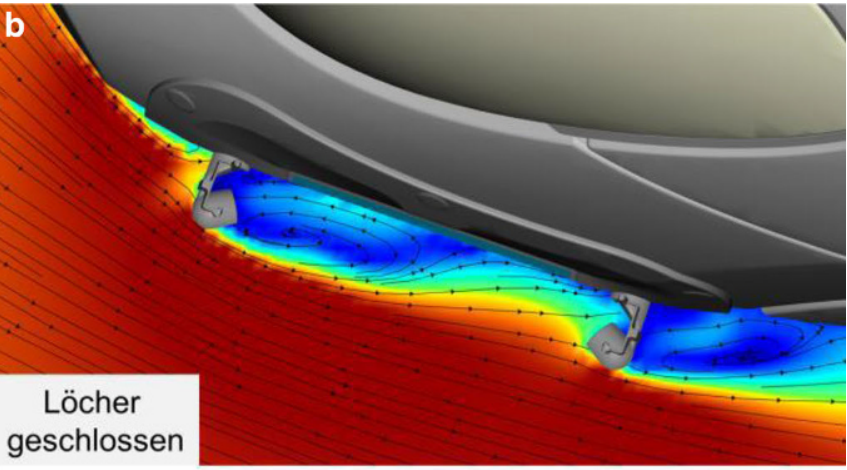

Dimensionslose Geschwindigkeitsmagnitude

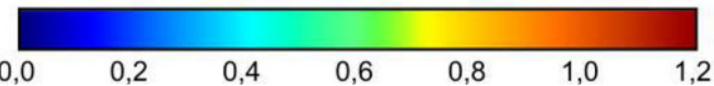

Abb. 14 Gemittelte, dimensionslose Geschwindigkeitsmagnitude mit Stromlinien in der Ebene der Reinigungsdüsen (a Ursprungszustand, b optimierte Variante)

darf gereinigt, da mehr Fluid die Sensorfläche erreicht. Die im Fahrzeug verbauten Reinigungsflüssigkeitsbehälter können kleiner dimensioniert werden, wodurch Gewicht und Kosten gespart werden können. Insgesamt weisen die Ergebnisse zur Simulation der Reinigungspartikel eine hohe Übereinstimmung mit den durchgeführten Experimenten im Umweltwindkanal und der Realerprobungen auf.

\section{Fazit}

Mehrphasensimulationen bieten ein enormes Potenzial zur frühzeitigen Analyse und Optimierung des externen Wassermanagements im Fahrzeugentwicklungsprozess, wie die drei aufgeführten Anwendungsfälle verdeutlichen. In allen Fällen wird eine sehr gute Übereinstimmung zwischen Experiment und Simulation erzielt. Der Einsatz solcher Simulationen wird in Zukunft weiter steigen, sodass selbst sehr komplexe Anwendungsfälle, wie bspw. die Sichtfrei- haltung bei hohen Fahrgeschwindigkeiten, zuverlässig und in angemessener Zeit simulativ untersucht werden können.

Funding Open Access funding enabled and organized by Projekt DEAL.

Open Access Dieser Artikel wird unter der Creative Commons Namensnennung 4.0 International Lizenz veröffentlicht, welche die Nutzung, Vervielfältigung, Bearbeitung, Verbreitung und Wiedergabe in jeglichem Medium und Format erlaubt, sofern Sie den/die ursprünglichen Autor(en) und die Quelle ordnungsgemäß nennen, einen Link zur Creative Commons Lizenz beifügen und angeben, ob Änderungen vorgenommen wurden.

Die in diesem Artikel enthaltenen Bilder und sonstiges Drittmaterial unterliegen ebenfalls der genannten Creative Commons Lizenz, sofern sich aus der Abbildungslegende nichts anderes ergibt. Sofern das betreffende Material nicht unter der genannten Creative Commons Lizenz steht und die betreffende Handlung nicht nach gesetzlichen Vorschriften erlaubt ist, ist für die oben aufgeführten Weiterverwendungen des Materials die Einwilligung des jeweiligen Rechteinhabers einzuholen.

Weitere Details zur Lizenz entnehmen Sie bitte der Lizenzinformation auf http://creativecommons.org/licenses/by/4.0/deed.de. 


\section{Literatur}

1. Demel D, Feldmann J, Schütz T, Tropea C (2019) Simulation der Regenwasserführung für eine bessere Sicht. Automobiltech Z 121:52-55. https://doi.org/10.1007/s35148-019-0144-6

2. Travin AK, Shur ML, Spalart PR, Strelets MK (2006) Improvement of delayed detached-eddy simulation for LES with wall modelling. In: European Conference on Computational Fluid Dynamics ECOMAS CFD Niederlande

3. Shur ML, Spalart PR, Strelets MK, Travin AK (2008) A hybrid RANS-LES approach with delayed-DES and wall-modelled LES capabilities. Int J Heat Fluid Flow 29(6):1638-1649. https://doi.org/ 10.1016/j.ijheatfluidflow.2008.07.001

4. Menter FR (1994) Two-equation eddy-viscosity turbulence models for engineering applications. AIAA J 32(8):1598-1605. https://doi. org/10.2514/3.12149

5. Meredith K, Heather A, De Vries J, Xin Y (2011) A numerical model for partially-wetted flow of thin liquid films. Comput Methods Multiph Flow VI:239-250. https://doi.org/10.2495/MPF110201

6. Gingold RA, Monaghan JJ (1977) Smoothed particle hydrodynamics: theory and application to non-spherical stars. Mon Not $\mathrm{R}$ Astron Soc. https://doi.org/10.1093/mnras/181.3.375

7. Liu AB, Mather D, Reitz R (1993) Modeling the effects of drop drag and breakup on fuel sprays. SAE Technical Paper 930072. https://doi.org/10.4271/930072

8. Hänel D (2004) Molekulare Gasdynamik. Einführung in die kinetische Theorie der Gase und Lattice-Boltzmann-Methoden. Springer, Berlin
9. Teixeira CM (1998) Incorporating turbulence models into the lattice-Boltzmann method. Int J Mod Phys 9(8):1159-1175. https:// doi.org/10.1142/S0129183198001060

10. Lambert JH (1760) Photometria, sive de mensura et gradibus luminis, colorum et umbrae. Sumptibus Vidae Eberhardi. Klett, Augsburg

11. Beer A (1852) Bestimmung der Absorption des rothen Lichts in farbigen Flüssigkeiten. Ann Phys Chem 86:78-88

12. Sylvain A, Bouchet J-P (2009) Quantitative assessment by UV fluorescence of rain water flow on vehicle body in the Jules Verne climatic wind tunnel. In: Wiedemann J (Hrsg) Progress in vehicle aerodynamics and thermal management: Proceedings of the 7th FKFS Conference. Expert, Renningen, S 127-142

13. BMW Group (2013) BMW Group Standard GS 97004-1. Kraftfahrzeugdichtheit gegen Wasser. Begriffe und Prüfungen

14. BMW Group (2013) BMW Group Standard GS 97004-2. Kraftfahrzeugdichtheit gegen Wasser. Anlagenspezifikation

15. KRÜSS GmbH Deutschland Mobile Surface Analyzer - MSA. https://www.kruss-scientific.com/files/kruss-bro-msa-de.pdf. Zugegriffen: 27. Mrz. 2021

16. Kölzer C (2019) Simulation des Abtropfverhaltens an geöffneten Fahrzeug-Heckklappen. Masterthesis, Technische Universität Kaiserslautern

17. Demel D (2021) Ein Beitrag zur Simulation der Sichtfreihaltung an Personenkraftwagen. Dissertation, Technische Universität Darmstadt 